\title{
Effects of Beam Startup Characteristics on Dose Delivery Accuracy at Low Monitor Units in Step-and-Shoot Intensity Modulated Radiation Therapy
}

\author{
Kazunori Fujimoto ${ }^{1,2}$, Kunihiko Tateoka ${ }^{1,3}$, Yuji Yaegashi ${ }^{1}$, Katsumi Shima ${ }^{1}$, Junji Suzuki ${ }^{1}$, \\ Yuichi Saito $^{1}$, Akihiro Nakata ${ }^{1}$, Takuya Nakazawa ${ }^{1}$, Tadanori Abe ${ }^{1}$, Masaki Yano ${ }^{1}$, \\ Masanori Someya $^{3}$, Kensei Nakata ${ }^{3}$, Masakazu Hori ${ }^{3}$, Masato Hareyama ${ }^{2}$, Koichi Sakata ${ }^{1,3}$ \\ ${ }^{1}$ Department of Medical Physics, Graduate School of Medicine, Sapporo Medical University, Sapporo, Japan \\ ${ }^{2}$ Radiation Therapy Research Institute, Social Medical Corporation Teishinkai, Sapporo, Japan \\ ${ }^{3}$ Department of Radiology, Sapporo Medical University, Sapporo, Japan \\ Email: tateoka@sapmed.ac.jp
}

Received September 11, 2012; revised October 15, 2012; accepted October 23, 2012

\begin{abstract}
Intensity modulated radiation therapy (IMRT) is a highly accurate technique that is usually implemented in either dynamic or step-and-shoot fashion with many segments each having low monitor units (MUs). The present study evaluated the effects of beam startup characteristics on the dose delivery accuracy for each segment at low MUs for step-and-shoot IMRT with an Elekta Precise accelerator at the highest dose rates. We used a two-dimensional semiconductor detector for the dose measurements. The field size of each segment was assumed to be $20 \times 20 \mathrm{~cm}^{2}$ and each segment was set to deliver 1 - 10 MUs. Our results show a variation in dose delivery accuracy between segments for the same IMRT beam, which can be attributed to the beam startup characteristics. This variability is attributed to the changes in the transient changes in the temperatures of the electron gun filament and the magnetron. That is, the transient increase in the temperature of the filament leads to increasing doses with time and that of the magnetron leads to decreasing doses with time during the first few MUs.
\end{abstract}

Keywords: Startup Performance; Step-and-Shoot IMRT; Low Monitor Units

\section{Introduction}

The goal of radiotherapy is to achieve tumor control by delivering a prescribed dose to a defined target volume, and to minimize delivered dose to neighboring normal tissue. Intensity modulated radiation therapy (IMRT) [1,2] is one popular method of achieving accurate dose delivery. IMRT is usually implemented using a multi-leaf collimator (MLC) in either the step-and-shoot or dynamic modes. In the latter mode, the intensity modulated beam forms irregularly shaped fields made by moving the MLC continuously during irradiation. In the former, multiple, small, irregularly shaped fields are created with the MLC (segments) and each can deliver an arbitrary number of monitor units (MUs), including low MUs ( $<10 \mathrm{MU})$. In both modes, it is necessary to deliver the correct dose for irregularly shaped fields made in the MLC. However, studies conducted on the beam characteristics at low MUs in IMRT have generally shown that the dose is nonlinear, nonuniform, and not reproducible at low MUs [3-7]. In particular, at low dose rates, the total beam-on time between the beam-on of the first segment and the beam-off of the last segment during IMRT becomes long- er than planned, leading to a somewhat low average dose rate. This has been found to affect the survival ratio of human cancer cells at different dose rates [8]. Moreover, a lower-than-planned dose rate leaves greater risk for repopulation of tumor cells as compared with acute irradiation, which favors the use of shorter irradiation times in IMRT by reducing the number of beams and segments through optimization of the number of subfields $[9,10]$. For step-and-shoot IMRT, therefore, it is important to adopt the highest possible dose rate that the linear accelerator can deliver.

Ezzell and Chungbin [3] reported that more MUs are delivered than planned with a radiation treatment planning system (RTPs) in the first segment of the IMRT beam when using a Varian accelerator; this is called the overshoot phenomenon. Likewise, Kang et al. [4] showed dose differences of up to $4 \%-5 \%$ can occur when using irradiation at less than $7 \mathrm{MU}$. These differences stem from the electronic delay in the control loop for the MLC and beam controller system, which are controlled by separate CPUs. Similarly, several reports have shown increased dose differences at low MUs in a segment of a 
step-and-shoot IMRT beam from accelerators manufactured by Philips, Siemens, and Elekta [5-7]. They showed that the cause of this dose difference was variation in the gun current or microwaves from the magnetron/klystron (high-power linear accelerators) during beam control (on/off) for segments in step-and-shoot IMRT. As a result, they were recommended use low dose rates as a solution to this problem.

In addition, for the Varian and Siemens linear accelerators, beam control is initiated by the grid pulse of the triode gun at high power settings. However, for the Elekta and Philips accelerators, only the first segment is initiated with pulses form the diode gun and microwaves from the magnetron at high power; from the second segment onward, the beam is initiated by the microwaves from the magnetron only. Moreover, some studies have shown a correlation between the arbitrary dose rate and the average dose delivered over three or five segments with the same low MU in step-and shoot IMRT. Finally, the beam startup characteristics could also affect dose at low MUs because of differences in beam control methods [11]. However, there have been no reported studies on the effects of beam startup characteristics on the dose delivery accuracy in each segment in step-and-shoot IMRT.

In this study, the effects of beam startup characteristics on the dose delivery accuracy for each segment at low MUs was investigated for step-and-shoot IMRT at the highest dose rate on an Elekta Precise accelerator (Precise Desktop 4.1, Elekta Ltd Crawley UK), the machine available at the author's institution.

\section{Materials and Methods}

Step-and-shoot IMRT beams having five segments were made by irradiating a virtual water equivalent phantom $\left(30 \times 30 \times 12.3 \mathrm{~cm}^{3}\right)$ using a treatment planning system (Pinnacle $^{3}$, Philips Radiation Oncology System, Madison, WI). The treatment plan data acquired from Pinnacle ${ }^{3}$ were imported to the Elekta Precise accelerator. The field size of each segment was set to $20 \times 20 \mathrm{~cm}^{2}$ with a source-to-surface distance (SSD) of $95 \mathrm{~cm}$ (i.e., sourceto-target distance of $100 \mathrm{~cm}$ ). Then, each segment was delivered perpendicularly to a 2D diode array detector (MapCheck, Sun Nuclear Corporation, Melbourne, USA) connected to a control computer. The MapCheck detector was placed between square water equivalent phantoms (Tough Water, Kyoto Kagaku Co., Ltd, Kyoto, Japan) with the dimensions $30 \times 30 \times 3 \mathrm{~cm}^{3}$ and $30 \times 30 \times 5$ $\mathrm{cm}^{3}$, the SSD was $95 \mathrm{~cm}$ (i.e., source-to-detector distance (SDD) of $100 \mathrm{~cm}$ ). The measurement depth was $5 \mathrm{~cm}$ water equivalent depth. This combination of water equivalent phantom and Mapcheck was similar to the treatment plan data from Pinnacle ${ }^{3}$. Dose calibration for the MapCheck detector was performed on an Elekta Pre- cise accelerator with $10-\mathrm{MV}$ photons, a field size of $20 \times$ $20 \mathrm{~cm}^{2}$, dose of $200 \mathrm{MU}$, SDD of $100 \mathrm{~cm}$, and measurement depth of $5 \mathrm{~cm}$ water.

All the measurements were performed for one fixed MU in each of the five segments, where the dosage was increased from 1 to $10 \mathrm{MUs}$ in steps of $1 \mathrm{MU}$ and then set to $200 \mathrm{MU}$. For each MU setting, five repeated measurements were acquired in a random sequence. Therefore, each segment was measured at each MU a total of five times and the total number of segments that delivered the same MU was 25. However, before the measurements, 500 MUs were dosed to avoid a systematic influence from the beam history (e.g., by warming of the electron gun). The measurements were performed with 10-MV photons at the maximum dose rate $(480 \mathrm{MU} / \mathrm{min})$ of the Elekta Precise accelerator.

Note that the dose of the Elekta Precise accelerator is checked daily with a square field $\left(10 \times 10 \mathrm{~cm}^{2}\right)$, which delivers about $2 \mathrm{~Gy}$ (corresponding to $200 \mathrm{MU}$ ), which is similar to the dose calibration method of all medical accelerators. Thus, the dose calibration factor of the monitor chamber of an Elekta Precise accelerator was used to deliver the correct dose.

In this report, the percent difference $(r)$ between the dose delivered in each segment $(x \mathrm{MU})$ and a dose of 200 MUs with a conventional radiation beam was determined for each measurement as follows:

$$
r(\%)=\left[\frac{\mathrm{D}(x \mathrm{MU})}{\mathrm{D}(200 \mathrm{MU})} \times \frac{200}{x}-1\right] \times 100
$$

where $\mathrm{D}(x \mathrm{MU})$ and $\mathrm{D}(200 \mathrm{MU})$ are the Mapcheck readings for $x \mathrm{MU}$ and $200 \mathrm{MU}$. A value of $r=0 \%$ is expected for a perfect dose. By using this equation, one can clearly see the percent difference of another MU.

\section{Results}

For comparison with previous results [3-7], we plotted the percent differences as a function of segment MU delivered for step-and-shoot IMRT with five identical segments and 10-MV X-ray photons at a dose rate of 480 $\mathrm{MU} / \mathrm{min}$ using the Elekta Precise accelerator (Figure 1). The tolerance of the X-ray monitor unit linearity of an IMRT has been reported to be $\pm 2 \%$ ( $\geq 5$ MUs) and $\pm 5 \%$ (2 - 4 MUs) in the AAPM TG-142 [12].The average difference was $-7.5 \%$ and the standard deviation was about $\pm 4 \%$ when 1 MU was delivered per segment. However, the average was within about $-4 \%$ and $-2 \%$ of the expected value when more than 2 MUs and 7 MUs were delivered per segment, respectively. Moreover, a decreasing trend can be seen in the average percent difference as the dose per segment increases. Note that all the differences were negative and the standard deviation was about $\pm 0.8 \%$ at 2 - 6 MUs per segment. Further, the av- 


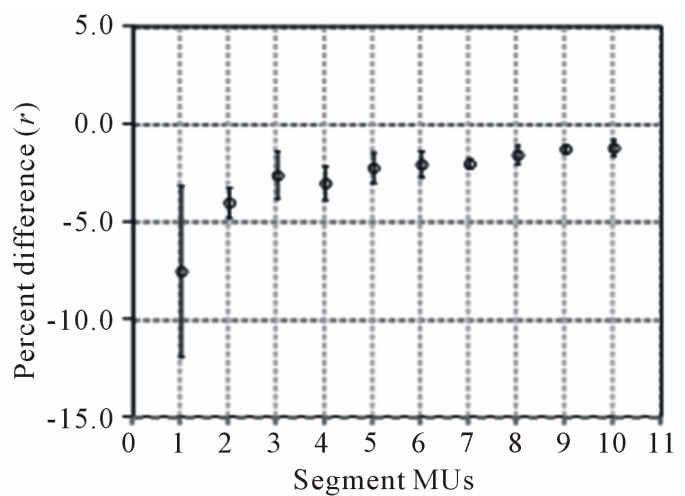

Figure 1. Percent difference $(r$ ) between the average dose delivered at various segment MUs and a 200 MU delivery. Symbols and error bars represent the averages and standard deviations of a total of $\mathbf{2 5}$ measurements.

erage percent difference and standard deviation were highest at $1 \mathrm{MU}$. These findings are consistent with those of previous publications [3-7].

Next, to analyze the effects of beam startup characteristic on dose accuracy at low MUs, we plotted the percent difference in each segment as a function of the dose, as shown in Figure 2. At $1 \mathrm{MU}$ per segment, the average percent difference was about $-1 \%$ in the first segment, but between $-7 \%$ and $-13 \%$ for the rest; that is, the first segment and other segments showed different trends. In addition, the percent difference in the first segment was within $-3 \%$ for all of doses, but decreased with an increase in the dose for the other segments, which is in close agreement with the results in Figure 1. The standard deviation is within about $\pm 5 \%$ for all of segments at $1 \mathrm{MU}$ per segment, but reduces to about $\pm 1.2 \%$ from 2 MU per segment. Thus, the first segment differs from the other segments possibly because of beam startup characteristics at low MUs in step-and-shoot IMRT. Furthermore, the percent differences in the second to the fifth segment were in close agreement for all doses.

To investigate the first segment further, the percent average difference of the first segment was compared against that of the other segments taken together as a function of segment MU (Figure 3). The discrepancy between the first and subsequent segments was the largest at $1 \mathrm{MU}$, but decreased to $3 \%$ at $2-6 \mathrm{MUs}$ and then to $1 \%$ at 7 MUs.

\section{Discussion}

Our results show that the percent average differences and standard deviations for all segments (a total of 25 measurements) were largest at $1 \mathrm{MU}$ per segment MU (Figure 1). This is consistent with previous reports on the Varian 21EX and Siemens PRIMUS accelerators [3,4,12]. For the former, Kang et al. [4] showed that the dose difference at low MUs could be attributed to the communica-

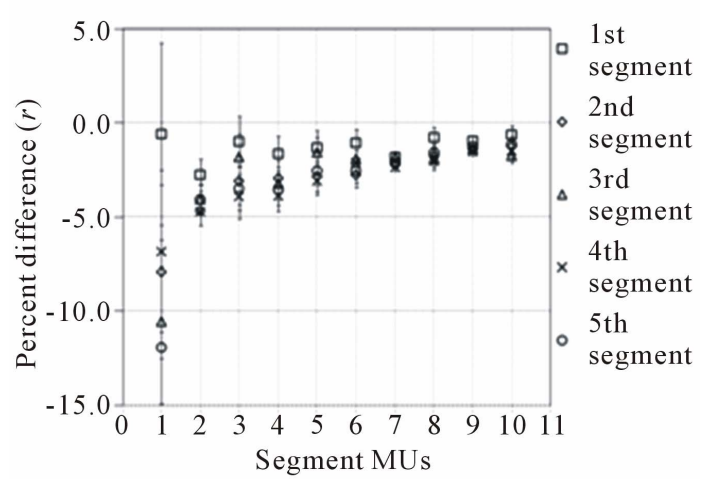

Figure 2. Percent difference ( $r$ ) between the average delivered dose at various segments MUs in each segment and a 200 MU delivery. Symbols and error bars represent the averages and standard deviations of a total of 5 measurements.

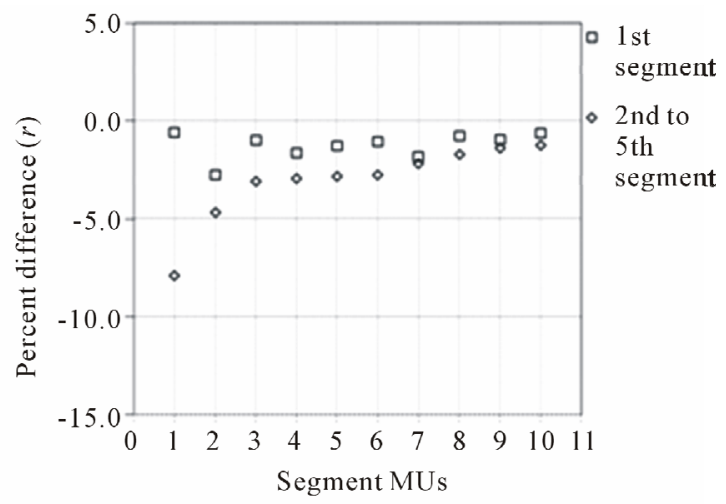

Figure 3. Percent difference $(r)$ between the average dose delivered at various segment MUs for the first segment and the second to the fifth segment relative to a $200 \mathrm{MU}$ delivery. Symbols and error bars represent the averages and standard deviations of a total of five measurements (for the first segment) and 20 measurements for second to the fifth segment.

tion delay between the separate beam controller and MLC controllers; the communication time is $50 \mathrm{~ms}$ for Varian linear accelerators [3]. For the latter, Saw et al. [12] reported similar behavior at less than $5 \mathrm{MU}$. They could be attributed to the time constant of the integration circuit of the monitor chamber, which is component-specific and regardless of the prescribe dose (planned MUs). This is because the integrated current from the monitor ionization chamber of the accelerator is used as a beam-off trigger. Similarly, the Elekta accelerator also has a significant time constant $(40 \mathrm{~ms})$ for the integration circuit of the monitor chamber, which could explain larger differences and standard deviations at $1 \mathrm{MU}$ per segment. However, the influence of the beam control technique on the dose delivered in each segment has not been previously investigated.

Next, as shown in Figures $\mathbf{2}$ and 3, we found that the 
percent difference can be expected to be within $-3 \%$ for all segment MUs in the first segment. However, it is nearly the same from the second to the fifth segment and shows a decrease with an increase in segment MU delivered. This result has not been previously reported. Mohr et al. [7] and Hansen et al. [13] indicated that beam quality may vary with time, especially during the first few MUs, which translates into variations in the beam quality factor $q$ and the depth-dose curve. However, they did not per- form a detailed analysis into why the beam energy varies with time during the first few MUs.

Here we consider two possible factors: the diode gun and the magnetron in high-power accelerators such as the Elekta Precise accelerator [14]. First, the X-ray beam of the Elekta Precise accelerator is turned on or off when a pulse voltage from the high-power linear accelerators is applied to the magnetron. However, this can cause rapid heating of the magnetron by eddy currents, which can affect its resonance structure. As a result, the resonant frequency of the magnetron decreases, as does the frequency of output microwaves. Therefore, the acceleration efficiency of electrons from the electron gun is decreased and the beam energy of the linear accelerator could show a slight reduction. The magnetron does include a metal tuner that must move into position to correct for these variations. However, it takes approximately $200 \mathrm{~ms}$ from the time the X-ray beam is turned on for the electron acceleration efficiency to recover to normal values.

Second, the filament of the electron gun is heated immediately after the beam is turned on. The temperature of the filament is slightly low just after the beam is turned on, which could result in somewhat fewer electrons (smalller beam current). As a result, the beam energy of the linear accelerator could slightly increase.

These two factors could explain the time variation, especially during the first few MUs and the change in the depth-dose curve, as described by Mohr et al. [7] and Hansen et al. [13]. The beam for the first segment is initiated by a pulse from the diode gun and the microwaves from the magnetron (high-power accelerators), but from the second segment onward, the beam is switched on and off based on the microwaves from the magnetron.

In this study, the time required to deliver $1 \mathrm{MU}$ was $125 \mathrm{~ms}$, which can be calculated from the dose rate of $480 \mathrm{MU} / \mathrm{min}$ and pulse rate frequency (PRF) of $400 \mathrm{~Hz}$ for the Elekta Precise accelerator. Therefore, when set to deliver $1 \mathrm{MU}$ per segment (125 ms), the beam energy in the first segment could increase slightly with time due to the influence the electron gun filament and could be decrease slightly with time due to the influence of the magnetron during the first few MUs. This could explain the small percent difference at $1 \mathrm{MU}$ per segment, as shown in Figure 3. In contrast, the beam energy in the second and subsequent segments at $1 \mathrm{MU}$ per segment
(125 ms) could decrease slightly with time due to the influence of the magnetron during the first few MUs. This explains the larger differences seen in Figure 3 at 1 MU per segment.

Next, at 2 MUs per segments ( $240 \mathrm{~ms})$, there may be sufficient time for the influences the filament and magnetron temperatures to reduce gradually (over several and several hundred milliseconds, respectively). This explains the small differences seen in Figure 3 for the first segment at 2 MUs per segment. Then, in the second and subsequent segments, the influence the magnetron temperature can also reduce gradually over several hundred milliseconds, which explains the reduced differences in the second and subsequent segments at 2 MUs per segment.

Regarding the fact that the impact of the beam start-up control is different for subsequent segments from the first segment of the step-and-shoot IMRT beam in the Elektalinac, which is focused in this study, from a com-parison of the dose of a low MU with the dose of the overall IMRT plan, the effect of the treatment plan is considered small.

In addition, the temperatures of the filament and magnetron are related to the flatness, symmetry, and homogeneity of IMRT beam. This represents is a far more complicated problem and needs further study. Further work is underway to clarify these relationships.

In conclusion, the study showed that the transient temperatures of the electron gun filament and magnetron, which determine to the beam startup characteristics, also affect the dose in each segment at low MUs in step-andshoot IMRT with the Elekta Precise accelerator. That is, the temperature change of the electron gun filament can increase the dose with time and that of the magnetron can decrease the dose with time during the first few MUs.

\section{Acknowledgments}

The authors are grateful to Dr. Jun Takada and Dr. Kenichi Tanaka of Department of Physics, Graduate school of Medicine, Sapporo Medical University for their extensive physics support. We appreciate the advice from the beginning to the end of this study from Dr. Kiyoshi Yoda of the medical physics division at ELEKTA Corporation. We also thank Dr. MiyakoMyoujin, a radiation oncologist at Keiyukai Sapporo Hospital, who understood the purpose of this study and cooperated willingly. In addition, we thank all the radiological technologists at Keiyukai Sapporo Hospital.

\section{REFERENCES}

[1] V. Y. Kuperman and W.-C. Lam, "Improving Delivery of Segments with Small MU in Step-and-Shoot IMRT," Medical Physics, Vol. 33, No. 4, 2006, pp. 1067-1073. 


\section{doi:10.1118/1.2174128}

[2] R. D. Wiersma and L. Xing "Examination of Geometric Accuracies of Gated Step-and-Shoot Intensity Modulated Radiation Therapy," Medical Physics, Vol. 34, No. 10, 2007, pp. 3962-3970. doi:10.1118/1.2776671

[3] G. A. Ezzell and S. Chungbin, "The Overshoot Phenomenon in Step-and-Shoot IMRT Delivery," Journal of Applied Clinical Medical Physics, Vol. 2, No. 3, 2001, pp. 138-148. doi:10.1120/1.1386508

[4] S.-K. Kang, K.-H. Cheong, T. Hwang, B. C. Cho, S. S. Kim, K. J. Kim, D. H. Oh and H. Bae, "Dosimetric Characteristics of Linear Accelerator Photon Beams with Small Monitor Unit Setting," Medical Physics, Vol. 35, No. 11, 2008, pp. 5172-5178. doi:10.1118/1.2995759

[5] V. N. Hansen, P. M. Evans, G. J. Budgell, J. H. L. Mott, P. C. Williams, M. J. P. Brugmans, F. W. Wittkämper, B. J. Mijnheer and K. Brown, "Quality Assurance of the Dose Delivery by Small Radiation Segments," Physics in Medicine and Biology, Vol. 43, No. 9, 1998, pp. 2665-2675. doi:10.1088/0031-9155/43/9/017

[6] M. Ravikumar, M. A. Al Asmary, R. A. Sultan and H. A. Al Ghamdi, "Dose Delivery Accuracy of Therapeutic Photon and Electron Beams at Low Monitor Unit Setting," Strahlentherapie und Onkologie, Vol. 181, No. 12, 2005, pp. 796-799. doi:10.1007/s00066-005-1412-9

[7] P. Mohr, S. Brieger, J. Stahl and G. Witucki, "Linearity of the Dose Monitor System at Low Monitor Units," Strahlentherapie und Onkologie, Vol. 183, No. 6, 2007, pp. 327-331. doi:10.1007/s00066-007-1596-2

[8] V. Moiseenko, C. Duzenli and R. E. Durand, "In Vitro Study of Cell Survival Following Dynamic MLC Inten-
sity-Modulated Radiation Therapy Dose Delivery," Medical Physics, Vol. 34, No. 4, 2007, pp. 1514-1520. doi:10.1118/1.2712044

[9] J. Z. Wang, X. A. Li, W. D. D'Souza and R. D. Stewart, "Impact of Prolonged Fraction Delivery Times on Tumor Control: A Note of Caution for Intensity-Modulated Radiation Therapy (IMRT)," International Journal of Radiation Oncology, Biology, Physics, Vol. 57, No. 2, 2003, pp. 543-552. doi:10.1016/S0360-3016(03)00499-1

[10] H. Paganetti, "Changes in Tumor Cell Response Due to Prolonged Dose Delivery Times in Fractionated Radiation Therapy," International Journal of Radiation Oncology, Biology, Physics, Vol. 63, No. 3, 2005, pp. 892900. doi:10.1016/j.ijrobp.2005.07.953

[11] M. Buchgeister and F. Nüsslin, "Startup Performance of the Traveling Wave versus Standing Wave Linear Accelerator," Medical Physics, Vol. 25, No. 4, 1998, pp. 493495. doi:10.1118/1.598225

[12] E. E. Klein, et al., "Task Group 142 Report: Quality Assurance of Medical Accelerator," Medical Physics, Vol. 36, No. 9, 2009, pp. 4197-4212. doi:10.1118/1.3190392

[13] C. B. Saw, S. Li, K. M. Ayyangar, M. Yoe-Sein, S. Pillai, C. A. Enke and J. C. Celi, "Dose Linearity and Uniformity of a Linear Accelerator Designed for Implementation of Multileaf Collimation System-Based Intensity Modulated Radiation Therapy," Medical Physics, Vol. 30, No. 8, 2003, pp. 2253-2256. doi:10.1118/1.1592640

[14] D. Greene and P. C. Williams, "Linear Accelerators for Radiation Therapy," 2nd Edition, Taylor \& Francis, New York, 1997. 